\title{
Research on automatic first break picking method in seismic data processing
}

\author{
Zhang Rongxi ${ }^{1, a}$ \\ ${ }^{a}$ College of Computer Science, Beijing University of Technology,Beijing, China \\ azhangrongxi@bjut.edu.cn
}

Keywords: EIFP; First arrival wave; automatic picking; first break picking

\begin{abstract}
The seismic data imaging and the first arrival pickup is an application of computer graphics and calculation method in the process of geological exploration. Through the computer technology the seismic data can be visualized, the first arrival wave can be picked rapid and accurately. It can help the geological professionals interpretate the seismic more accurately. This paper puts forward a wave picking method which will save the labor's pickup time, improve the picking precision and develop the EIFP system (seismic data imaging and fist arrival picking initial system).
\end{abstract}

\section{Introduction}

Picking up first arrival time is the first step work of processing of seismic exploration data. The accuracy of first arrival time reflects the quality of acquisition work, on the other hand, it is also one of the most important factors of processing quality of seismic data. Ensure accurate picking the first arrival time is to do a very important work in seismic exploration. When the seismic wave arrive at some observation points, the point particle medium began to vibrate and the moment called the first arrival wave. The characteristics of primary wave with the take-off time most early, strong energy. In theory, before the first arrival the seismic wave amplitude should be zero. In the actual acquisition, various kinds of interference will influence the data, such as mobile phone signal, high voltage lines, which makes its waveform distortion. When the SNR is high, the first arrival is obvious, easy to identify; when SNR is low, the first arrival is not obvious, is not easy to identify.

In the technical constraints, the original seismic first arrival pickup is picking through the artificial manual. Hand picking exist two disadvantages: one is the workload is very large, because of a work area often have hundreds or even thousands of road, to pick them becomes a time-consuming, labor consuming and complicated work; the other is the personal experience related to the level of technology, especially in the complex area, artificial are often difficult to get the first arrival time accurately. In the development of Mathematical Science, electronic technology and information technology, the seismic wave first break picking methods appeared.

\section{Overview of the EIFP system}

Now the seismic processing software in the market usually for a data format such as .SEGY or .CST format, and there is not a software which put these two kinds of data into integration. So EIFP system using these two kinds of data formats, and provides convenience for the treatment of different types of files.

First, it mapped the seismic data to the screen coordinate file, make the seismic data are shown in graphs; and then select a new first picking algorithm; Finally, put the picking up method in the real seismic data, and make a detailed comparison with other methods, show correctness of the selected algorithm.

In order to analyze the seismic profile better, the EIFP system increase the normalized energy function based on the needs of the user. Concrete structure system is shown in Fig 1. 


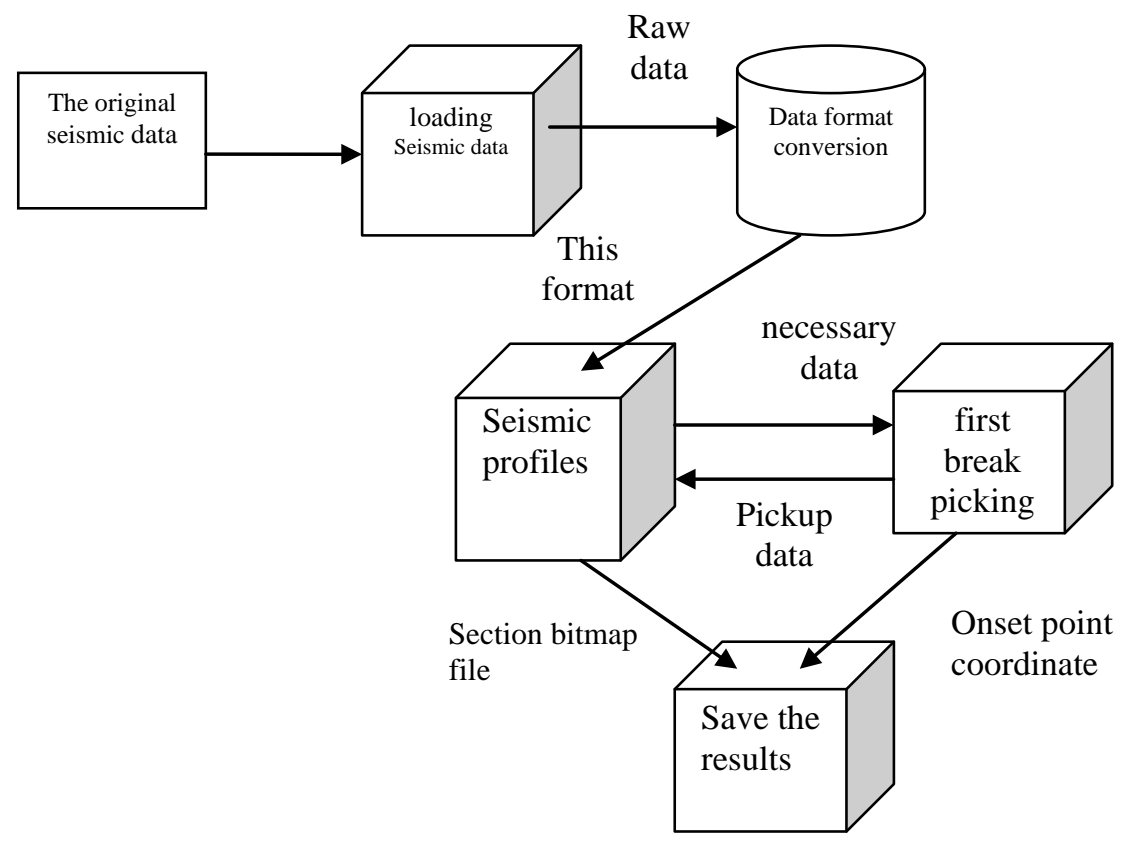

Fig 1. EIFP system structure diagram.

\section{Study on pick up algorithm}

People usually according to the change of amplitude, frequency or phase of seismic signal to determine the first arrival time, also some according to the correlation of adjacent channel signals to judge the beginning time. So far, many method to automatically pick up first arrival data have been proposed, such as the correlation method and the energy ratio method and fractal dimension method and neural network method etc..

The current seismic first arrival automatic picking method can be divided into the following four categories:

1) method based on the instantaneous characteristics of seismic records, and its characteristic is with clear physical meaning, simple operation and is very suitable at high SNR, but the disadvantage is that when SNR is low, the beginning to feature is not obvious adn not easy to pick up.

2) According to the method of cross-correlation to determine the initial to adjacent channel, has certain inhibition on the noise, but by the early to "continued to" great influence for surface wave, the result will be poor in complex area.

3) The artificial intelligence method to comprehensive earthquake wave dimension information, such as neural network method, this kind of method has very good effect to suppress noise, with respect to accuracy in picking up the first arrival, but the method's implementation is complex and low efficiency.

4) The method based on edge detection algorithm performs well in low noise, but it's sensitive to noise which nearby the first arrival. The algorithm will fail when the interference is strong.

Table1. Automatic first break picking method comparison

\begin{tabular}{|l|l|l|l|}
\hline $\begin{array}{l}\text { Automatic first break } \\
\text { picking method }\end{array}$ & $\begin{array}{l}\text { Attribute key to the realization } \\
\text { characteristics }\end{array}$ & scope of application \\
\hline Correlation method & $\begin{array}{l}\text { correlation between } \\
\text { the seismic trace }\end{array}$ & The first channel & $\begin{array}{l}\text { Seismic data topographic } \\
\text { change uniform }\end{array}$ \\
\hline Energy ratio method & $\begin{array}{l}\text { Window energy } \\
\text { before and after }\end{array}$ & $\begin{array}{l}\text { length of time } \\
\text { window } \\
\text { sigh signal to noise ratio of } \\
\text { seismic data }\end{array}$ \\
\hline Edge detection method & $\begin{array}{l}\text { Image detection } \\
\text { detection }\end{array}$ & $\begin{array}{l}\text { edge now data } \\
\text { lgorithm }\end{array}$ \\
\hline
\end{tabular}


As can be seen from the table, different first break picking method on the basis of the seismic attributes and characteristics for picking up the first arrivals. Energy ratio method using first arrival's before and after energy, correlation method on the basis of correlation characteristics between seismic trace. First break picking different methods have the certain range, the existing method has a certain limitation in practical application, not ideal for picking up all cases first, therefore in the process of picking up the first arrivals on seismic data, to consider the data characteristics, using automatic picking suitable method. So after a comprehensive comparison decided to adopt the energy ratio method of first break picking.

As long as there is enough signal-to-noise ratio, the above first break picking methods will get ideal results, but in the low SNR case, the pick accuracy of some first picking method are not ideal. Therefore need a set of advanced technology as support to the extent, greatly reduce the labor intensity, and improve the accuracy of automatic first break picking time. "The seismic data imaging and the first arrival pickup system (EIFP system) is put forward in this background.

\section{Improvement of the energy ratio method}

1. Analysis of energy ratio method

1) Advantages

A method is proposed, and has a long-term application in practical work to illustrate that this method has certain advantage. Since energy ratio method was proposed in 1985, has been in the application of picking up the first arrivals of seismic exploration. Now we analyze the characteristics of the method.

(1)From the definition we can see energy ratio method with clear physical meaning, using the energy characteristics of seismic waveform to judge on the first arrival time, the definition of simple, easy to realize on the computer.

(2) Compared with other methods, the energy ratio method of automatic first break picking speed is faster than picked up manually, fractal dimension method and neural network method. For the seismic data of first breaking strong energy area, the energy ratio method is very practical.

2) Shortcomings

When picking first arrival of seismic data, the seismic data types can be classified into two categories: the data with high signal-to-noise ratio and initial seismic data to clear and are affected by topography and other factors of low SNR, the weak first arrival seismic data. For the first kind of seismic data, several commonly used methods are all well determine the first arrival time. But the fractal method has some difficulty in step and choose of interpolation, and its algorithm, in particular are less accurate in judging the "V" shape; the neural network method can accurately pick up seismic data, the learning process is complicated and slow, the use of computer processing speed is slow. We can see that the energy ratio method in the implementation process and theories, has the advantages of simple algorithm, short computation time, but the energy ratio method also has some shortcomings.

(1)The seismic data with low signal-to-noise ratio, or amplitude of seismic data in the early arrival signal lower than the subsequent wave and noise, energy ratio method can not accurately picking the first arrival time;

(2) When using energy ratio method to pick first break, the choice of the time window length have great influence on the first arrival pickup.

(3) For the weak first arrival, energy ratio method in the window, after the energy ratio is not obvious, the judgment is not accurate for the first arrival time;

(4) Because of the magnitude of a few spots above onset point may have some value close to zero, the formula denominator tends to zero, the $\mathrm{R}$ will be singularity.

2. Improvement of the energy ratio method

For a set of seismic data, their cycles may be different, but in the use of energy ratio method, the length of time window can be set. But once set the window length, it can not be arbitrarily changed. We found that problem is the amplitude of a few samples of each initial close to zero, to make $\mathrm{R}$ 
produce singular. So we decided to use a new pickup method from the energy ratio method which be improved in three aspects, to improve the accuracy of first arrival pickup.

(1)Before the seismic wave arrive the amplitude value become zero, by increasing the coefficient method to make the energy ratio of no singularity;

(2)Through an improved energy to replace the amplitude ratio of energy, so that can accomplish the goal when the signals are poor, to identified the first breaking time accurately.

(3)The improvement is usually static window division method, a dynamic model of the front and rear window energy ratio, so that the moving window dynamically, to ensure that the arrival time in the time window midpoint.

In order to avoid $\mathrm{R}$ to be singular, formula is amended as:

$$
\begin{gathered}
R=\frac{\left[\sum_{t=T_{0}}^{T_{2}} A^{2}(t)\right]^{\frac{1}{2}}+\alpha}{\left[\sum_{t=T_{1}}^{T_{0}} A^{2}(t)\right]^{\frac{1}{2}}+\alpha} \\
\alpha=\left[\sum_{t=0}^{T} A^{2}(t)\right]^{\frac{1}{2}} / N
\end{gathered}
$$

$\alpha$ is a seismic trace relative energy, $\mathrm{N}$ is used in seismic trace points. Eq (1) is improved by using the seismic instantaneous attributes, get the energy ratio of the envelope waveform.

Usually based on the traditional energy ratio method for picking up the first arrivals, the seismic trace from the head to tail is divided into many a time window, and then calculate the energy ratio. Because of the first to arrive one after another, if static partitioning time window, may make the energy ratio of the window is not the greatest. Window rolling method, through the rolling windows can calculate the energy ratio in the window many times to determine the first arrival position. It can greatly improve the accuracy and stability of the first breaking time.

Time window rolling start from the head to tail, circulating a window. Because it is a cycle time window after repeat moving form, so it's only need to move a window length. Moving a calculated each energy ratio, and choose the maximum value, and then find out the maximum value from these selected energy ratio, then the maximum seismic records at the time is corresponding to the onset point.

The specific steps are as follows:

(1)Take the seismic channel into a plurality of small time window, window length should be as small as possible;

(2) Calculate energy ratio between begin and after time window;

(3) Select the maximum energy ratio

(4) Increase a time window length respectively, and repeat 1 - 3 step;

(5)Find out the maximum value, and the value of the corresponding time points, namely for the first arrival time.

Respectively using energy ratio, improved energy ratio method of a set of data for picking up the first arrivals, according to the manual pick up early to get higher accuracy, it can be used as the other two methods of the standard, and comparison of two kinds of methods, shown in table 2.

Table 2. Processing data of three methods

\begin{tabular}{|l|l|l|l|l|l|}
\hline Trace & $\begin{array}{l}\text { Hand } \\
\text { picking }\end{array}$ & Energy ratio & Absolute error & $\begin{array}{l}\text { Improvement of } \\
\text { the energy ratio }\end{array}$ & Absolute error \\
\hline 1 & 55 & 54 & 1 & 56 & 1 \\
\hline 2 & 64 & 62 & 2 & 64 & 0 \\
\hline 3 & 66 & 65 & 1 & 67 & 1 \\
\hline 4 & 69 & 72 & 3 & 68 & 1 \\
\hline 5 & 74 & 71 & 3 & 75 & 1 \\
\hline
\end{tabular}




\begin{tabular}{|l|l|l|l|l|l|}
\hline 6 & 77 & 74 & 3 & 78 & 1 \\
\hline 7 & 79 & 81 & 3 & 80 & 1 \\
\hline 8 & 82 & 84 & 2 & 82 & 0 \\
\hline 9 & 88 & 86 & 2 & 88 & 1 \\
\hline 10 & 94 & 93 & 1 & 94 & 1 \\
\hline 11 & 97 & 99 & 2 & 97 & 0 \\
\hline 12 & 102 & 109 & 7 & 100 & 2 \\
\hline 13 & 103 & 110 & 7 & 105 & 2 \\
\hline 14 & 110 & 112 & 2 & 112 & 2 \\
\hline 15 & 115 & 116 & 1 & 116 & 1 \\
\hline 16 & 118 & 121 & 3 & 120 & 2 \\
\hline 17 & 122 & 117 & 5 & 123 & 1 \\
\hline 18 & 126 & 124 & 2 & 128 & 2 \\
\hline 19 & 130 & 133 & 3 & 134 & 4 \\
\hline 20 & 132 & 135 & 3 & 134 & 2 \\
\hline 21 & 138 & 142 & 4 & 140 & 2 \\
\hline 22 & 148 & 146 & 2 & 149 & 1 \\
\hline average absolute error & & 3 & & 1.4 \\
\hline
\end{tabular}

\section{Automatic pick test}

Click the automatic button, the system will be based on improved energy ratio method calculate the first arrival time, and then according to the coordinates to redraw profile. Taking into account that when the paper is printing these red points cannot be displayed, so in the test, the maximum amplitude is set to smaller, this profile only shows strong energy, makes the onset point more clearly. Click the automatic pick up button, then the program will be get the Y coordinate of each first arrival, and finally displayed on the screen. As shown in Fig2.

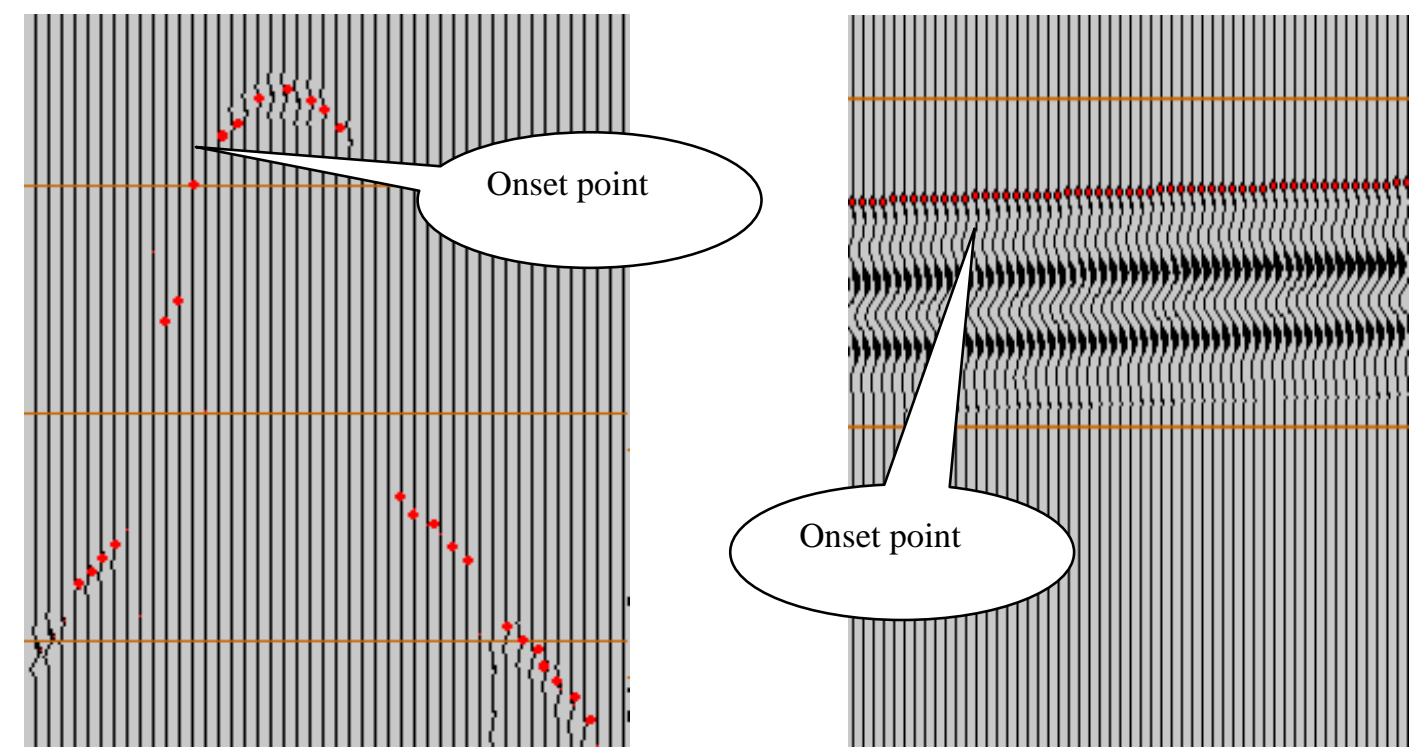

Fig 2.cst first arrival pickup diagram

Fig 3.segy first arrival pickup diagram

\section{Conclusions}

Based on the improved energy ratio method in theoretical research, proves that it has certain feasibility in theory; through the analysis and calculation on front of the actual data, the improved energy ratio method in the automatic first break picking seismic data has achieved very good results, confirmed the validity of the improvement of the energy ratio method. 
(1)According to the actual seismic data, can be seen from the table, improvement of the energy ratio method in picking up the first arrivals, the maximum absolute error is the smallest, and the average absolute error is minimized, the accuracy is the highest, it proves that the improved energy ratio algorithm is stability;

(2)The improvement of the energy ratio algorithm because of the rolling window method, in picking up the first arrivals without considering the time window length selection, reducing the workload;

(3) In the multichannel seismic data processing, improved energy ratio method, because of its simple formula, can quickly realized in the computer.

The realization of EIFP system will be greatly increased the efficiency of seismic exploration processing: originally needs a lot of manpower several days to complete the workload now only a few hours, or even a few minutes to complete, greatly saves the project costs, but also the picking precision can be improved significantly.

\section{Acknowledgements}

This work was supported by the "rixin talent" cultivation project of Being University of Technology.(Grant No.007000543114532).

\section{References}

[1] F.Coppens. First arrival picking on common-offset trace collections for automatic estimation of static corrections[J],Geophysical Prospecting,1985, 6(1), 33 : 1212-1231.

[2] Gelchinsky, Shtirelman. Automatic picking of first arrival and parameterization oftravel-time curves, Geophysical Prospection[J], 1983, 15(2) : 55-60

[3] Pan Shulin, Gao Lei, Zhou bustling. A method to realize the first picking [J]. geophysical prospecting for petroleum, 2005, 44 (2): 163-166.

[4] Pan Shulin, Gao Lei, Zhou bustling. Picking [J].2006 to single track boundary detection and spline interpolation based on the primary, 45 (3): 425-430

[5] Zhuang Donghai, Xu Yun, Wuda bala. Seismic time window attribute feature detection of first arrival of [J]. Journal of Jianghan Petroleum Institute, 1999, 21 (4): 45-48

[6] Zuo Guoping, Wang Yanchun, Sui Rongliang. An improved method of [J]. geophysical prospecting for petroleum, energy utilization ratio of seismic first arrival pickup 2004, 43 (4): 345-347 